\title{
Molecular characterisation of a versatile peroxidase from a Bjerkandera strain
}

\author{
Patrícia R. Moreira ${ }^{a, b}$, C. Duez ${ }^{c}$, D. Dehareng ${ }^{c}$, A. Antunes ${ }^{d}$, \\ E. Almeida-Vara ${ }^{a}$, J.M. Frère ${ }^{c}$, F. Xavier Malcata ${ }^{b}$, J.C. Duarte $^{a}, *$ \\ a Departamento de Biotecnologia, Instituto Nacional de Engenharia, Tecnologia e Inovação (INETI), \\ Est. Paço do Lumiar, 22, P-1649-038 Lisboa, Portugal \\ ${ }^{\mathrm{b}}$ Escola Superior de Biotecnologia, Universidade Católica Portuguesa, R. Dr. António Bernardino de Almeida, \\ P-4200-072 Porto, Portugal \\ c Centre d'Ingénierie des Protéines, Université de Liège, Institut de Chimie, B6, Sart-Tilman, B-4000 Liège, Belgium \\ ${ }^{\mathrm{d}}$ Département de Chimie Générale et Physique, Laboratoire de Spectrométrie de Masse, Université de Liège, \\ B6a, Sart-Tilman, B-4000 Liège, Belgium
}

Received 7 January 2005; received in revised form 2 May 2005; accepted 12 May 2005

\begin{abstract}
The cloning and sequencing of the rbpa gene coding for a versatile peroxidase from a novel Bjerkandera strain is hereby reported. The $1777 \mathrm{bp}$ isolated fragment contained a $1698 \mathrm{bp}$ peroxidase-encoding gene, interrupted by 11 introns. The 367 amino acid-deduced sequence includes a 27 amino acid-signal peptide. The molecular model, built via homology modelling with crystal structures of four fungal peroxidases, highlighted the amino acid residues putatively involved in manganese binding and aromatic substrate oxidation. The potential heme pocket residues (R44, F47, H48, E79, N85, H177, F194 and D239) include both distal and proximal histidines (H48 and H177). RBP possesses potential calcium-binding residues (D49, G67, D69, S71, S178, D195, T197, I200 and D202) and eight cysteine residues (C3, C15, C16, C35, C121, C250, C286, C316). In addition, RBP includes residues involved in substrate oxidation: three acidic residues (E37, E41 and D183) - putatively involved in manganese binding and $\mathrm{H} 83$ and W172 - potentially involved in oxidation of aromatic substrates. Characterisation of nucleotide and amino acid sequences include RBP in versatile peroxidase group sharing catalytic properties of both $\mathrm{LiP}$ and MnP. In addition, the RBP enzyme appears to be closely related with the ligninolytic peroxidases from the Trametes versicolor strain.
\end{abstract}

() 2005 Published by Elsevier B.V.

Keywords: Ligninolytic peroxidases; White-rot fungi; Cloning and sequencing; Tertiary structure; Introns; rbpa gene

\footnotetext{
* Corresponding author. Tel.: +351 217165 141; fax: +351217163636.

E-mail address: jose.duarte@ineti.pt (J.C. Duarte).
}

\section{Introduction}

During the latest decade, research on the lignindegradation ability of fungi has mainly focused on 
a few basidiomycetes known as white-rot fungi. The complexity of the lignin-degradation mechanisms, which encompass different enzymes (depending on the fungus), has prompted isolation of novel fungal strains-in attempts to find and characterise new enzymes, regarding reaction mechanism and substrate preference.

A novel class of ligninolytic peroxidases, named versatile peroxidases, with high affinity for manganese and dyes, has been described; these enzymes can also oxidise 2,6-dimethoxyphenol (DMP) and veratryl alcohol (VA) in a manganese-independent reaction (Camarero et al., 1996, 1999, 2000; Giardina et al., 2000; Heinfling et al., 1998a,b,c; Martinez, 2002; Mester and Field, 1998; Palma et al., 2000; Ruiz-Duenas et al., 1999b, 2001). Until now, however, those enzymes have only been isolated from Pleurotus ostreatus, Pleurotus eryngii, Pleurotus pulmonarius, Bjerkandera adusta and Bjerkandera sp. strain BOS55.

The recently sequenced enzymes MnPL1 and MnPL2 from cultures of $P$. eryngii exhibit high sequence and structural similarities with LiP from Phanerochaete chrysosporium; however, molecular models show a putative manganese interaction site near the internal propionate moiety of heme. The presence of this binding site is essential for direct oxidation of $\mathrm{Mn}^{2+}$ (Ruiz-Duenas et al., 1999a).

MnP isoenzymes, purified from P. ostreatus and duly characterised by Giardina et al. (2000), are able to oxidise phenolic substrates both in the presence and absence of manganese, as already reported (Giardina et al., 2000; Heinfling et al., 1998b; Mester and Field, 1998 ) for isozymes produced by other strains of $P$. ostreatus, P. pulmonarius, P. eryngii, B. adusta and Bjerkandera sp.

The catalytic properties of versatile peroxidases from both Pleurotus and Bjerkandera spp. are similar to each other and differ from those of LiP and MnP; these differences in catalytic properties, as well as in structural characteristics (Camarero et al., 1999; RuizDuenas et al., 2001), justify the description of such versatile peroxidases as part of a new peroxidase family in class II (fungal) peroxidases (Martinez, 2002).

A fungal strain, which exhibits high decolourisation activities on poly R-478 and Remazol Brilliant Blue R (RBBR) dyes, was collected from rotting lignocellulosic material; this novel fungal strain was iden- tified in our laboratory as belonging to the Bjerkandera genus and was tentatively named Bjerkandera sp. strain $B 33 / 3$. Analysis of peroxidase activities in the extracellular fluid of said strain demonstrated the existence of lignin peroxidase, as well as manganese-dependent and manganese-independent peroxidase activities (Moreira et al., 2001). Several peaks were obtained after MonoQ ion-exchange chromatography of the extracellular fluid, which were characterised by distinct enzyme activities. One of the main peaks associated with the dye decolourising activity exhibited also properties characteristic of the novel class of versatile peroxidases, with ability to oxidise manganese, as well as VA and DMP in a manganese-independent reaction. This new enzyme was named RBP.

The objectives of the present study were to clone the RBP-encoding gene and to characterise both the enzyme and its gene-encoding structures.

\section{Materials and methods}

\subsection{Performance of culturing}

Bjerkandera sp. strain B33/3 was grown in CDBYE medium, as described previously in detail (Moreira et al., 2001).

\subsection{Determination of proteinaceous features}

From the extracellular fluid of the Bjerkandera sp. B33/3 strain, the main enzyme responsible for the dye decolourisation was isolated and purified following resolution by Mono-Q ion-exchange chromatography. This enzyme, named RBP is able to oxidise manganese, as well as veratryl alcohol and 2,6-dimethoxyphenol in a manganese-independent reaction; hence, it can be included in the new group of versatile ligninolytic peroxidases. Oxidation of DMP was estimated by measuring the absorbance at $469 \mathrm{~nm}\left(30^{\circ} \mathrm{C}\right)$, during oxidation of $1 \mathrm{mM}$ DMP in $50 \mathrm{mM}$ sodium malonate buffer ( $\mathrm{pH} 3.0$ and 5.0), in the presence of $1 \mathrm{mM}$ ethylene diamine tetra-acetic acid (EDTA). Oxidation of veratryl alcohol was determined by measuring absorbance at $310 \mathrm{~nm}\left(30^{\circ} \mathrm{C}\right)$, during the oxidation of $4.0 \mathrm{mM}$ veratryl alcohol in $100 \mathrm{mM}$ sodium tartrate buffer ( $\mathrm{pH} 3.0$ and 5.0). The RBBR decolourising activity was assayed spectrophotometrically by measuring the 
decrease in absorbance at $595 \mathrm{~nm}\left(30^{\circ} \mathrm{C}\right)$. The enzymatic standard reaction mixture consisted of $0.05 \mathrm{mM}$

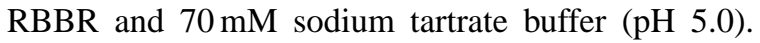
The manganese(II) activity was assayed spectrophotometrically by measuring the decrease in absorbance at $238 \mathrm{~nm}\left(30^{\circ} \mathrm{C}\right)$. The enzymatic standard reaction mixture consisted of $0.1 \mathrm{mM} \mathrm{MnSO}_{4}$ and $100 \mathrm{mM}$ sodium tartrate buffer ( $\mathrm{pH}$ 5.0). The 2,2'-azinobis(3ethylbenzothiazoline-6-sulphonic acid) diammonium salt (ABTS) oxidising activity was assayed spectrophotometrically by measuring the decrease in absorbance at $420 \mathrm{~nm}\left(25^{\circ} \mathrm{C}\right)$. The enzymatic standard reaction mixture consisted of $0.5 \mathrm{mM}$ ABTS and $100 \mathrm{mM}$ sodium tartrate buffer ( $\mathrm{pH}$ 5.0). Kinetic constants were determined for several substrates. The RBP enzyme in stake presents high affinity for (the oxidising substrate) manganese at $\mathrm{pH} 5.0$, with $K_{\mathrm{m}}$ values $(96 \mu \mathrm{M})$. Small values of $K_{\mathrm{m}}$ for DMP oxidation in the absence of manganese were also obtained $(66$ and $99 \mu \mathrm{M})$ depending at the $\mathrm{pH}$ tested, 3.0 and 5.0, respectively. The smallest $K_{\mathrm{m}}$ values were obtained were recorded for oxidation of RBBR and ABTS $(2 \mu \mathrm{M}$ for both); this realisation is consistent with the hypothesis that RBP is a versatile peroxidase with high affinity for dye substrates, e.g. anthraquinone-derived and high redox compounds, e.g. ABTS (which are substrates usually preferred by plant peroxidases). Oxidation of VA is favoured at $\mathrm{pH} 3.0$, as happens with LiP peroxidases and has an apparent $K_{\mathrm{m}}$ value of $3400 \mu \mathrm{M}$.

Purified native protein $(200 \mu \mathrm{g})$ was N-deglycosylated overnight at $\mathrm{pH} 5.5$ with $80 \mathrm{mU}$ of endoglycosidase $\mathrm{H}$ from Roche Molecular Biochemicals (Mannhein, Germany). SDS-PAGE of native and deglycosylated proteins was performed on PhastGel $^{\circledR}$ high-density polyacrylamide gels (Amersham Pharmacia Biotech, Uppsala, Sweden). The gel was calibrated using the low molecular weight calibration kit (Amersham Pharmacia Biotech) as standard.

Isoelectric focusing of purified native protein was performed in PhastGel ${ }^{\circledR}$ (Amersham Pharmacia Biotech) IEF polyacrylamide gels, with a $\mathrm{pH}$ range of 3.0-9.0. The gel was calibrated using the low $\mathrm{p} I$ calibration kit (Amersham Pharmacia Biotech) as standard.

Protein bands were stained with Coomassie Brilliant Blue R-250.

Protein mass was also estimated by ESI-MS/MS using a Q-Tof-2 ${ }^{\mathrm{TM}}$ mass spectrometer (Micromass,
Manchester, UK) using ca. $25 \mu \mathrm{g}$ of purified native protein.

\subsection{Sequencing of tryptic peptides}

The purified native protein was digested via addition of 1:20 (w/w) of a $0.1 \mu g \mu l^{-1}$ solution of trypsin (Roche Molecular Biochemicals), reconstituted in $1 \mathrm{mM} \mathrm{HCl}$. In order to accelerate digestion, $1 \%(\mathrm{v} / \mathrm{v})$ acetonitrile was added to a $500 \mathrm{mM}$ ammonium acetate buffer (pH 7.4) containing $20 \mathrm{mM} \mathrm{CaCl}_{2}$, which was incubated for $12 \mathrm{~h}$ at $37^{\circ} \mathrm{C}$. The tryptic peptides were fractionated and desalted by elution on a ZipTip ${ }_{\mathrm{C} 18}$ pipette tip (Millipore, Billerica, USA). Elution was carried out with mixtures of water/acetonitrile/acetic acid: 93/5/2, 78/20/2 and 48/50/2 (v/v/v), respectively. The fractions obtained were analysed by nano-ESI-MS/MS using a Q-Tof $-2^{\mathrm{TM}}$ mass spectrometer (Micromass) as described by Shevchenko et al. (2000). Selection of the ions analysed and adjustment of the collision energy were made manually. Peptides were delivered to the mass spectrometer by silica capillaries obtained from Protana (MDS Proteomics, Odense, Denmark). The BioLynx software (Micromass) was used for the prediction of MS/MS fragmentation patterns from peptide sequences and comparison with existing mass spectra. The fragmentation data obtained were analysed using the PepSeq sequencing software (Micromass). Database searches using BLAST 2.1.3 (NCBI http server) programs (Altschul et al., 1997), were performed with the sequences obtained, in order to eliminate those resulting from trypsin autodigestion.

\subsection{Extraction of DNA and RNA}

DNA and RNA were extracted from a 6-day-old mycelium, which was collected by filtration, frozen with liquid $\mathrm{N}_{2}$ and ground to powder form with a mortar-and-pestle. For the DNA extraction, the cell powder was suspended in $10 \mathrm{mM}$ Tris- $\mathrm{HCl}$ buffer $(\mathrm{pH}$ 7.6) containing $1 \mathrm{mM}$ EDTA (TE), homogenised in a guanidinium thiocyanate and $N$-lauroylsarcosine denaturing suspension according to Pitcher et al. (1989) and incubated for $2 \mathrm{~h}$ in ice; $10 \mathrm{M}$ ammonium acetate was then added and after a further $10 \mathrm{~min}$ of incubation, the total DNA was extracted with 24:1 (v/v) chloroform/isoamyl alcohol. The mixture was centrifuged, the aqueous phase was transferred to another tube and 
0.8 volume of isopropanol was added. The DNA was washed with $70 \%$ (v/v) ethanol and the resulting pellet was air-dried and redissolved in TE buffer. The mixture was treated with RNase $\left(50 \mu \mathrm{g} \mathrm{ml}^{-1}\right)$ for $30 \mathrm{~min}$ at $37^{\circ} \mathrm{C}$ and extracted once again with $24: 1(\mathrm{v} / \mathrm{v})$ chloroform/isoamyl alcohol. The supernatant was recovered and supplemented with $1: 10(\mathrm{v} / \mathrm{v}) 3 \mathrm{M}$ sodium acetate (pH 5.2) and 2.5 volume of cold $\left(-20^{\circ} \mathrm{C}\right)$ absolute ethanol. After centrifugation for $5 \mathrm{~min}$ at $3000 \times \mathrm{g}$, the pellet was rinsed with $70 \%(\mathrm{v} / \mathrm{v})$ ethanol, air-dried and redissolved in TE. The quality and purity of DNA was evaluated spectrophotometrically at 260 and $280 \mathrm{~nm}$ and by electrophoresis in TAE buffer on $1.5 \%$ agarose gel stained with ethidium bromide.

The RNA isolation from Bjerkandera sp. B33/3 was performed with the SV total RNA isolation system (Promega Corporation, Madison, WI, USA) according to the manufacturer's instructions. The quality and purity of the total RNA was evaluated spectrophotometrically.

\subsection{Performance of $P C R$}

Escherichia coli Top10F', XL1-Blue or DH5 $\alpha$ were used for cloning recombinant plasmids. The polymerase chain reaction (PCR) products were cloned into the pGEM-T easy vector (Promega Corporation).

The oligonucleotides were purchased from Eurogentec (Liège, Belgium) or from Amersham Pharmacia Biotech. The PCR amplifications were performed with biotools DNA polymerase (Biotechnological and Medical Laboratories, Madrid, Spain). For the cloning of fragments adjacent to a known sequence, the LA-PCR in vitro cloning kit was used according to the supplier's instructions of the (Takara Shuzo, Shiga, Japan).

\subsection{Synthesis of $c D N A$}

The cDNA was prepared from the total RNA with ImProm-II $^{\mathrm{TM}}$ reverse transcription system (Promega Corporation), with small modifications of the manufacturer's protocol. The reverse transcription step was initiated with primer NTAG1 (5'-GCAGTGGTAACAACTTTTTTTTTTTTMM-3') on 0.08 or $0.8 \mu \mathrm{g}$ of total RNA in the buffer supplied, supplemented with $3 \mathrm{mM} \mathrm{MgCl} 2$, in the presence or absence of Sequencer ${ }_{X}$ Enhancer Solution F (Invitrogen, Carlsbad, USA). The annealing temperature was $37^{\circ} \mathrm{C}$.
PCR amplification of the cDNA was obtained using NTAG1 and F-peroxi (5'-TGCCCCGACGGCGTIAACACC-3') primers. F-Peroxi was synthesised on the basis of the N-terminal sequence of the purified peroxidase (VAXPDGVNTA) assuming that the nonidentified residue in the sequence determined was cysteine, a highly conserved residue at the third position of mature lignin peroxidases. Sequencer ${ }_{X}$ Enhancer Solution $\mathrm{F}$ was added to the reaction mixture. The annealing temperature was $39^{\circ} \mathrm{C}$ in the first $3 \mathrm{PCR}$ cycles and $50^{\circ} \mathrm{C}$ in the next 22 cycles.

The quality of the cDNA was evaluated by electrophoresis on a $1.0 \%$ agarose gel. The PCR products (without further purification) were shotgun cloned into the pGem- $\mathrm{T}$ easy vector according to the manufacturer's instructions.

In parallel, the PCR products of interesting sizes were purified from agarose gels with the geneclean spin kit (Qbiogene, Illkirch, France). A 3'-A overhang was added to the purified PCR fragments with the Biotools DNA polymerase, before cloning them into the pGemT easy plasmid.

The ligation products were used to transform $E$. coli DH5 $\alpha$ competent cells. Colonies were screened by PCR with M13 universal and reverse- 40 primers. Clones with inserts of expected size were transferred to $2 \times$ YT liquid medium, supplemented with $50 \mu \mathrm{g} \mathrm{ml}^{-1}$ ampicillin and grown overnight at $37^{\circ} \mathrm{C}$. Plasmids were isolated with the GFX Micro Plasmid Prep Kit (Amersham Pharmacia Biotech) according to the manufacturer's instructions and digested with EcoRI to estimate the size of the inserts by electrophoresis.

\subsection{Sequencing of DNA}

Sequencing reactions were carried out using the AutoRead sequencing kit (Amersham Pharmacia Biotech), with the fluorescent (cy5) M13 Universal and Reverse primers, SP6, T7 or specific internal oligonucleotides. Electrophoresis was done with an ALF Express II DNA sequencer (Amersham Pharmacia Biotech). The PSI-BLAST 2.2.8 at NCBI http server (Altschul et al., 1997) and the BioEdit Sequence Alignment Editor (Hall, 1999) programs were used for analysis, alignment and comparison of sequences of amino acids, DNA and cDNA sequences.

A dendogram based on similarities between sequences of mature peroxidases was obtained using 
the UPGMA method (after bootstrap of sequences), from PAM distance matrix of progressive pairwise multiple sequence alignment using the BioEdit Sequence Alignment Editor and phylogenetic inference package (PHYLIP) program (Felsenstein, 1993). LiP, MnP, VP and CIP, as well as other peroxidases from Bjerkandera sp. strain B33/3 (B33/3), B. adusta (BA), P. ostreatus (PO), P. eryngii (PE), Ceriporiopsis subvermispora (CS), Ganoderma applanatum (GA), Dichomitus squalens (DS), Coprinus cinereus (CC), Lepista irina (LI), Phlebia radiate (PR), Trametes versicolor (TV) and P. chrysosporium (PC) were compared to one another. The GenBank accession numbers (gene or cDNA sequences) are as follows: B33/3-RBP, AY217015; PE-MnPL1, AF007221; PE-PS1, AF175710; PO-MnP, POU21878; PO-MnP2, POS243977; PO-MnP3, AB011546; CSMnP1, AF013257; CS-MnP2A, AF161078; CSMnP3, AF161585; GA-MnP1, AB035734; DSMnP1, AF157474; DS-MnP2, AF157475; LI-VPS1, CAD56164; CC-CIP, X70789; PR-LigIII, P20010; PR-MnP2, CAC85963; PR-MnP3, CAC84573; TVMP2, Z30668; TV-PGVII, Z54279; TV-PGV, X77154; TV-CVMNP, D86493; TV-LP7, Z30667; TV-LPGII, X75655; TV-LiP12, M64993; TV-VLG1, M55294; TV-MRP, AF008585; PC-MnP1, M60672; PC-MnP2, L29039; PC-MnP3, U70998; PC-LiPA (H8), M27401; PC-LiPB (H8), M37701; PC-LiPC, M63496; PCLiPD (H2), X15599; PC-LiPE (H8), M92644; PCLiPF, M77508; PC-LiPH (H8), M24082; PC-LiPJ, AF140062.

\subsection{Modelling of proteinaceous structure}

Molecular modelling of the Bjerkandera sp. RBP versatile peroxidase (devoid of signal peptide) was performed by sequence homology using the PROMODII program (Guex and Peitsch, 1997; Peitsch, 1995, 1996). It was based on alignment of four fungal peroxidases, for which crystal models are available that present the largest similarities at the sequence level: LiP-H2 (Broohaven PDB entries 1QPAA: 66.35\% identity and 1QPAB: 66.35\% identity); LiP-H8 (1LLP: 64.75\% identity); MnP1 (1MNP: $53.58 \%$ identity) from $P$. chrysosporium. The complete enzyme, with its heme moiety, two calcium ions and surrounding water molecules, was built by superposition with the A chain of QPA peroxidase (1QPA) with the program
InsightII (Biosym Technologies, 1993a). The geometry of the whole system was optimised at the molecular mechanics (MM) level (Burkert and Allinger, 1982) with Amber force field (Weiner and Kollman, 1981; Weiner et al., 1986) using the program Discover (Biosym Technologies, 1993b) on a SGI Indy workstation. A dielectric constant equal to 2 was chosen and the threshold for convergence was selected as maximum force fixed at $0.02 \mathrm{kcal} \mathrm{mol}^{-1} \AA^{-1}$.

The Swiss-PDB Viewer v3.7 SP5 (Guex and Peitsch, 1997) and Rasmol v2.7.2.1 (Bernstein, 2003) programs were used for model comparison and presentation.

\section{Results}

\subsection{Determination of proteinaceous features}

The mass of native and deglycosylated RBP, determined with SDS-PAGE on PhastGel ${ }^{\circledR}$ high-density polyacrilamide gels were ca. 45.7 and $43.2 \mathrm{kDa}$, respectively, indicating that the mass of non-proteinaceus residues amounts to $5.6 \%$ of the whole molecular mass.

The mass of the RBP, which was also estimated by ESI-MS/MS, is ca. $37 \mathrm{kDa}$.

The isoelectric point estimated by gradient PAGE is ca. 3.5.

\subsection{Sequencing of tryptic peptides}

Sequencing of two internal peptides generated from RBP via tryptic digestion was possible using mass spectrometry, two positive ESI $\mathrm{m} / \mathrm{z}$ spectrum were obtained. The sequences obtained were DSVTDILNR and LQSDADFAR. The first peptide presented a theoretical $\mathrm{m} / \mathrm{z}$ of 516.7702 for a measured value of $516.7416 \mathrm{~m} / \mathrm{z}$, thus presenting a delta mass of $-55 \mathrm{ppm}$. The second peptide presented a theoretical $\mathrm{m} / \mathrm{z}$ of 511.7493 for a measured value of $511.7228 \mathrm{~m} / \mathrm{z}$, thus presenting a delta mass of $-52 \mathrm{ppm}$.

\subsection{Synthesis and cloning of rbpa cDNA}

The 1139 bp cDNA sequence (GenBank AF490538) corresponded to a single open reading frame. As expected, the deduced amino acid sequence of this fragment contained both the (previously identified) DSVTDILNR and LQSDADFAR internal tryptic peptides. 


\subsection{Cloning of rbpa gene}

With the purpose of cloning the gene encoding for RBP, the oligonucleotides F-peroxi $\left(5^{\prime}\right.$-TGCCCCGACGGCGTIAACACC- $\left.3^{\prime}\right)$ and Cterm6 (5'CACAATTCCTACGACGACGCCTTATTCCCTCC$3^{\prime}$ ) - complementary to the $3^{\prime}$ end of the cDNA sequence, were used in a PCR experiment pertaining to the total genomic DNA. A 1625 bp fragment was amplified and cloned. Six different templates were completely sequenced on both strands using internal specific oligonucleotides as primers. The Bestfit program from GCG Wisconsin package (Accelrys, San Diego CA, USA) clearly identified the correspondence between the amplified fragment and the cDNA and allowed accurate localisation of the introns: 10 short introns interrupted the ORF encoding the mature protein.

Since the F-peroxi primer encodes seven amino acids beginning at the third residue of the mature protein $\mathrm{N}$-terminus, the amplified fragment was devoid of the $5^{\prime}$ end of the gene. In order to obtain it, the oligonucleotide Nterm2 (5'-GCACTTCTTCGCCGCACTCGCCGCCGTC-3'), directed towards the $5^{\prime}$ end of the gene, was combined with an hexamer (random priming) in a PCR reaction with the total genomic DNA; the PCR products were cloned into the pGEM-T easy vector and to improve the specificity of amplification, a second PCR was performed on the ligation mixtures-with the Nterm3 oligonucleotide (5'-GTTCTGTTGGATGTCATCACGGACGGCG-3') and the M13 Universal or Reverse primer. One clone contained an ORF, with a good score of similarity with the signal peptides of manganese or lignin peroxidases.

The pair of oligonucleotides, $6 \mathrm{~h}$ complementary to the sequence encoding the peptide DDIEPNFHANN (5'-CGTTGTTGGCGTGGAAGTTGGGCTCGATGTCGTC- $\left.3^{\prime}\right)$ and PS1 (5'-ATGGCCTTCAAGCAACTCCTCACTG- $3^{\prime}$ ), allowed the expected signal peptide to be amplified. No promoter region was obtained for this gene.

The sequence of the $1771 \mathrm{bp}$ DNA fragment (Fig. 1) isolated during this research effort received the Accession no. AY217015 in the GenBank database.

Referring to the GT-AG rule of the intron splice sequences and to the homology between this DNA fragment and the cDNA previously sequenced, it was possible to predict the intron/exon structure of the rbpa gene. The 11 introns (for a total of $597 \mathrm{bp}$ ), with an average length of $54 \mathrm{bp}$ and their processing sequences are highlighted in Fig. 1. The translation initiation and termination codons, as well as a polyadenylation signal on the flanking $3^{\prime}$ region and the amplification primer locations are also indicated.

The intron splice junction sequences strictly adhere to the GT-AG rule and all of the putative internal Lariat sites conform to the NNHTNAY rule.

A schematic representation of the number, size and position of introns in the rbpa gene, as compared with those of related genes encoding fungal peroxidases from $B$. adusta, T. versicolor, $P$. ostreatus, $P$. eryngii and $P$. chrysosporium, is depicted in Fig. 2. The rbpa open reading frame has a $58 \% \mathrm{G}+\mathrm{C}$ content $(62 \%$ for the first, $48 \%$ for the second and $65 \%$ for the third position, respectively); a much lower (42\%) G + C content was found in the intron sequences.

The 367 amino acid-deduced sequence (including a 27 amino acid-signal peptide) is provided in Fig. 3. The deduced molecular mass of the mature protein is $35.7 \mathrm{kDa}$ and its (theoretical) isoelectric point is 4.3 . $\mathrm{RBP}$ does not contain any tyrosine residue; this is characteristic of most ligninolytic peroxidases. Tyrosine residue presence would otherwise result in oxidation of the enzyme (Camarero et al., 2000).

The sequence includes one potential $\mathrm{N}$-glycosylation site (N103) according to the NXS/T consensus, which could account for the $5.6 \% \mathrm{~N}$-linked carbohydrate.

The deduced heme pocket residues (R44, F47, H48, E79, N85, H177, F194 and D239), that include both distal and proximal histidines (H48 and H177), are also highlighted in Fig. 3.

RBP possesses potential calcium-binding residues (D49, G67, D69, S71, S178, D195, T197, I200 and D202) and eight cysteine residues (C3, C15, C16, C35, C121, C250, C286, C316), which are characteristics also shared by other fungal peroxidases (Camarero et al., 2000).

In addition, RBP includes residues involved in substrate oxidation, three acidic residues (E37, E41 and D183) - putatively involved in manganese binding and H83 and W172-potentially involved in oxidation of aromatic substrates (Banci, 1997; Martinez, 2002; Ruiz-Duenas et al., 2001).

The sequence relationship between 38 selected fungal (mature) peroxidases is illustrated in the dendogram 
ATGGCCTTCAAGCAACTCCTCACTGCTGCTCTTTCCATCGCCCTTG

Ps1

CTCTCCCCTTCTCGCAAGgttegtatctgttgetgeagaccgtattctectcgatgctcacttt Intron I

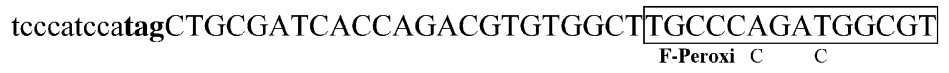

GAACACCGCAACCAACGCAGCCTGTTGTGCTTTGTTCGCCGTCCG TGATGACATCCAACAGAACTTGTTCGACGGCGGCGAGTGCGGCG AAGAAGTGCACGAGTCACTTCGACTgtatggattttgtaccetcgttgtgaattctgcg Intron II ctcattctctacccttagGACCTTCCACGATGCTATTGGCATATCTCCAAGC CTTGCCGCCACTGGCAAATTCGGgtacatattgggactttatttgtataatcgtggcgac Intron III taacgactgttacttagCGGCGGAGGTGCCGACGGGTCTATCATGATCTTC GACGACATCGAGCCCAACTTCCACGCCAACAACGGCGTGGACGA GATTATCAACGCGCAGAAGCCCTTCGTGGCCAAGCACAACATGA CGGCAGGTGACTTgtaagttgctgtttactccettagtgatattacgttcgetaaatcacacaaac

Intron IV

agTATTCAATTCGCAGGCGCCGTTGGCGTGAGCAACTGCCCTGGT GCTCCTCAACTGAGCTTCTTCCTCGGGCGCCCTGCAGgtacggaatttgc aacgattggatgttcttactctcac cetgcttgtagCGACGCAGCCCGCGCCTGACGGG Intron $\mathrm{V}$

CTTGTTCCGGAGCCCTTCGgtcagtccaattattgtcecgaatcettgcactcggactgatg Intron VI atgttcatcagACTCGGTCACCGACATCCTCAATCGCTTTGCCGATGCT GGCGGCTTCACAATTCAAGAAGTTGTTTGGCTCCTTGCCTCgtaagta ctaccetgggctegctaagtttegggtcctaattgatgatcatgtceatagTCATTCCATTGCTG

Intron VII

CTGCTGACCACGTCGACCCGACGATCCCTGGATCACCCTTCGATT CTACTCCCGAAATCTTCGACACACAGTTCTTTGTTGAGACGTTGT TGAAGGGCACGTTGTTCCCAGGgtaaacetcgtaaccatacttttaatcatggaagatg 6c

ctaatttaattatgatetagTACGAGCGGCAACCAGGGCGAAGTCGAGTCCC CACTTGCCGGCGAAATTCGTCTCCAGTCAGATGCCGACTTCGCAC GTGACTCGAGGACT GCTTGCGAGTGGCAGTCTTTCGTCAgtgagtactt 6h

tctatacaaaagaaaaacaggcatttatacgatcgectcagATAACCAGCCCCGGATGCA

Fig. 1. Nucleotide sequence of the rbpa gene from Bjerkandera sp. strain B33/3. The polyadenylation signal AATAA in the 3'-flanking region, as well as the translation initiation ATG and termination TAA codons are indicated in underlined bold characters. The 11 deduced introns are indicated in lower case and the intron processing sequences ( $5^{\prime}$ - and $3^{\prime}$-splicing and Lariat sites) are indicated in bold. The boxes denote the location of some important primers used for cloning the gene. 
Intron IX

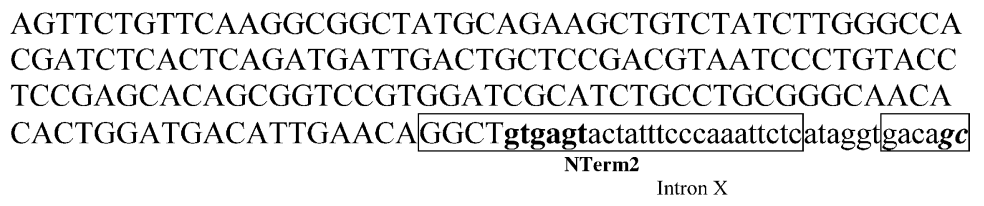
$\frac{\text { ctaacgtaagatatagTGCGCCT }_{\text {NTerm }}}{\text { CCACGCCATTCCCCTCGCTCACCGCCGA }}$

CCCTGGTCCGGCCACCTCTGTTGCCCCTGTgtaagtctcaacaatacgatatggt tgagcgeccecatctgac actactgatagCCCGCCTTCGTAAACGCTTCAAGCAA

Intron XI Stop

TGGAGTGATTGGGTTCTCACGACTCGGA $\frac{\text { GGGAATAAGGCGTCGT }}{\frac{\text { CTerm6 }}{4}}$

CGTAGGAATTGTG

Fig. 1. (Continued).

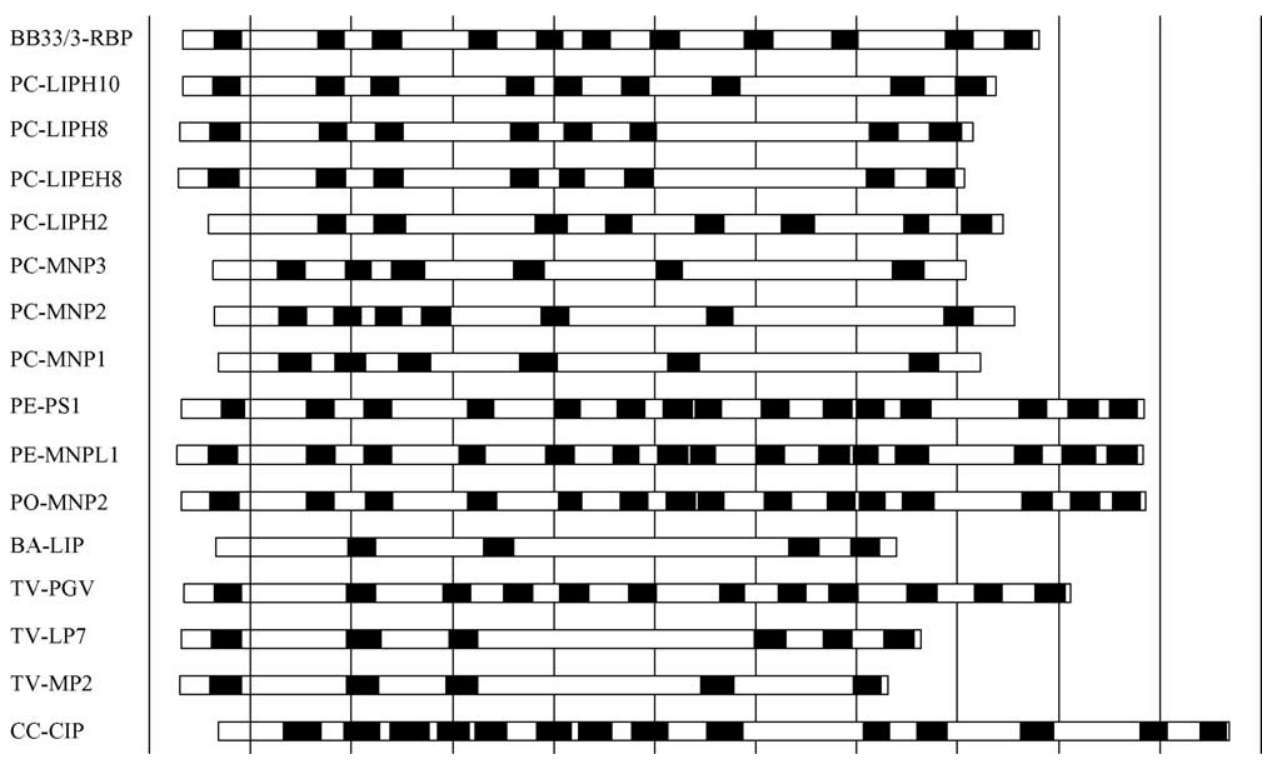

Fig. 2. Positions of exons (white) and introns (black) in genes encoding various LiP, MnP and other peroxidases. The second vertical line corresponds to the beginning of the mature protein. The distance between every line corresponds to 200 nucleotides. (1) $C$. cinereus peroxidase (GenBank no. X707892); (2) T. versicolor manganese peroxidase isoenzyme MP2 (GenBank no. Z30668); (3) T. versicolor lignin peroxidase isoenzyme LP7 (GenBank no. Z30667); (4) T. versicolor peroxidase PGV (GenBank no. X77154); (5) B. adusta lignin peroxidase (GenBank no. E03952); (6) P. ostreatus manganese peroxidase MnP2 (GenBank no. AJ243977); (7) P. eryngii polyvalent peroxidase MnPL1 (GenBank no. AF007224); (8) P. eryngii versatile peroxidase PS1 (GenBank no. AF175710); (9) P. chrysosporium manganese peroxidase MnP1 (GenBank no. M77513); (10) P. chrysosporium manganese peroxidase MnP2 (GenBank no. L29039); (11) P. chrysosporium manganese peroxidase MnP3 (GenBank no. U70998); (12) P. chrysosporium lignin peroxidase LiPDH2 (GenBank no. X15599); (13) P. chrysosporium lignin peroxidase LiPEH8 (GenBank no. M92644); (14) P. chrysosporium lignin peroxidase LiPH8 (GenBank no. M27884); (15) P. chrysosporium lignin peroxidase LiPCH10 (GenBank no. M63496); (16) Bjerkandera sp. strain B33/3 RBP peroxidase (GenBank no. AY217015). 


\begin{tabular}{|c|c|c|c|c|}
\hline MAFKQLLTAA & LSIALALPFS & QAAITRRVAC $\underline{\mathrm{C}}$ & PDGVNTATNA & ACCALFAVRD \\
\hline DIQQNLFDGG & ECGGEEVHESL & ㄴLTHDAIGI & SPSLAATGKF & 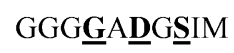 \\
\hline FDDIE्ENFE్ & ANNGVDEIIN & AQKPFVAKHNㅗ & $\underline{\text { MTA }}$ & GAVGVSN $\underline{\text { CPG }}$ \\
\hline APQLSFFLGR & PAATQPAPDG & LVPEPE्FDST & DILNRFADAG & 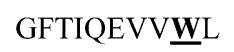 \\
\hline LAS $\underline{\text { HSIAAA }}$ & HVDPTIPGSP & $\underline{\text { FDSTPEIFDT }}$ & QFFVETLLKG & TLFPGTSGNQ \\
\hline GEVESPLAGE & IRLQSDEA & RDSRTAㅌEWQ & SFVNNQPRMQ & VLFKA \\
\hline SILGHDLTQM & IDCSDVIPVP & PSTAVRGSHL & PAGNTLDDIE & QA $\underline{\text { CASTPFPS }}$ \\
\hline TADPGPATS & VAPVPPS & & & \\
\hline
\end{tabular}

Fig. 3. Amino acid sequence of the RBP versatile peroxidase from Bjerkandera sp. strain B33/3 deduced from the rbpa gene. The 367 amino acid-deduced sequence includes a 27 amino acid-signal peptide containing a protease-splicing sequence (shown in bold and italic). The underlined bold letters indicate a putative $\mathrm{N}$-glycosylation residue (NMT), the eight cysteine residues, the heme pocket residues (R44, F47, H48, E79, N85, H177, F194 and D239) that include both distal and proximal histidines (H48 and H177, respectively), the potential calcium binding residues (D49, G67, D69, S71, S178, D195, I197, I200 and D202) and the residues involved in substrate oxidation (E37, E41 and D183 for manganese and H83, F149, W172 and A240 for aromatic substrates).

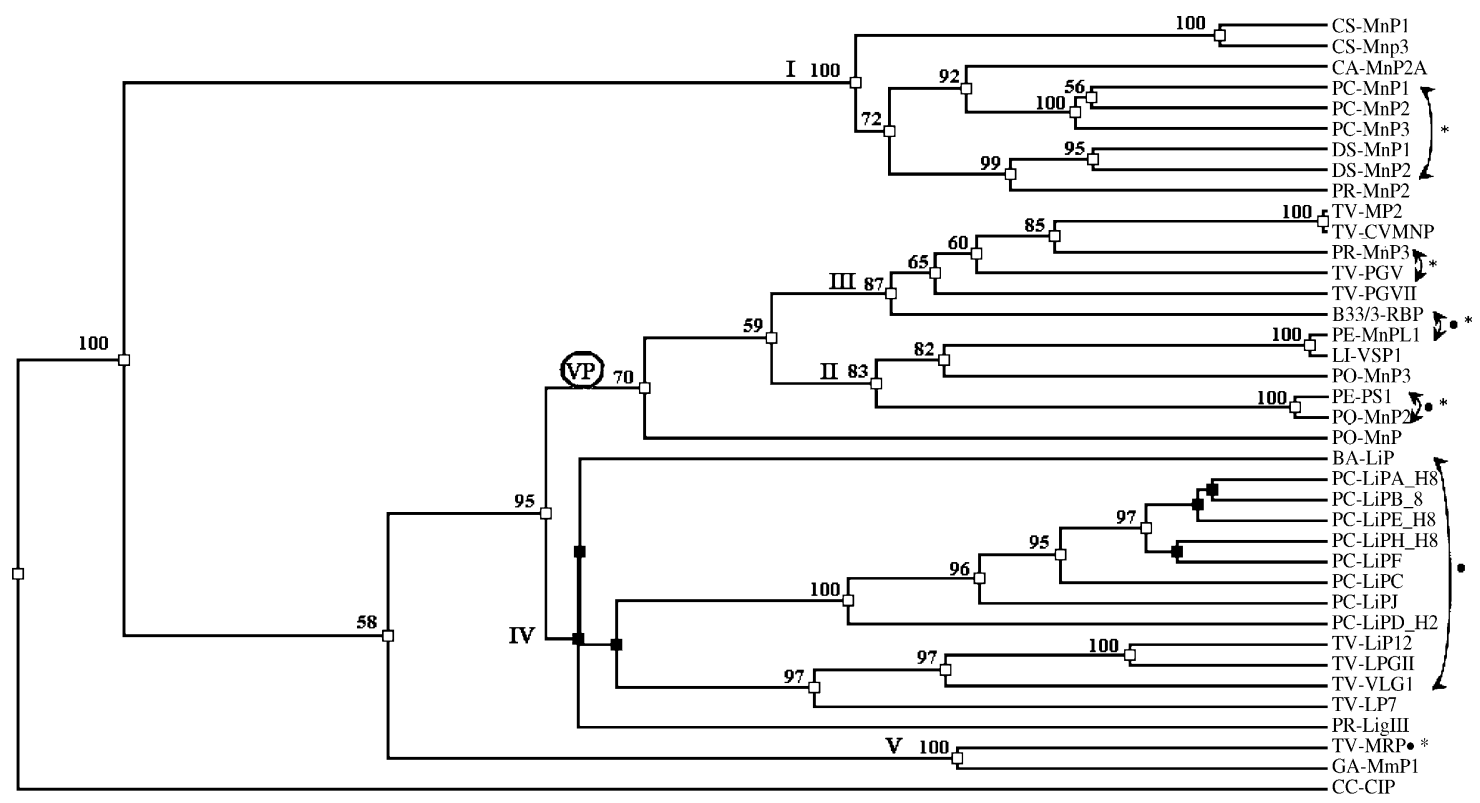

$\underline{0.01}$

Fig. 4. Phylogenetic relationship between mature fungal peroxidases. The dendogram was built from PAM distances between mature proteins using the PHYLIP software package after multiple alignment of 38 fungal peroxidase sequences and using the UPGMA method. The presence of manganese binding site $(*)$ and of exposed tryptophan involved in VA oxidation $(\bullet)$ are duly indicated. Nodes are represented as squares and bootstrap values of more than $50 \%$ (as percentage) are represented as ( $\square$ ). Nodes with bootstrap values of less than $50 \%$ are represented as black squares. 


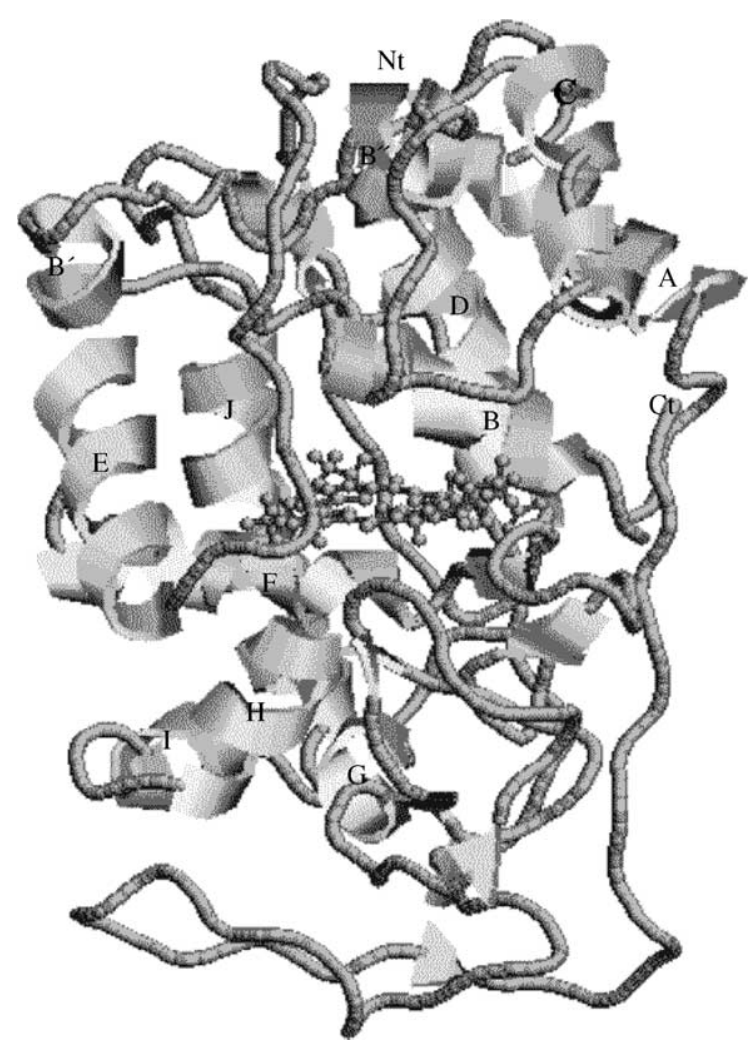

Fig. 5. Schematic molecular representation of the RBP peroxidase from Bjerkandera strain B33/3, after homology modelling with PROMODII using LiP and MnP crystal models as templates and refinement by Discover. The positions of $\alpha$-helices (A-J) and of the $\mathrm{C}$-termini $(\mathrm{Ct})$ and $\mathrm{N}$-termini $(\mathrm{Nt})$ are duly indicated.

in Fig. 4. Our dataset produced a single, fully resolved dendogram, but bootstrap support for some nodes is weak.

\subsection{Modelling of proteinaceous structure}

A three-dimensional model for the mature RBP was obtained by homology modelling, as made available in Fig. 5; this molecular model includes $12 \alpha$-helices, named according to the cytochrome $C$ peroxidase nomenclature (Finzel et al., 1984), viz.: helices A (A14-I25), B (V39-A50), B' (P55-P59), $\mathrm{B}^{\prime \prime}$ (S71-I74), C (E90-H102), D (A106-S119), E (V152-A162), F (I167-H177), G (Q204-T209), H (Q237-R244), I (A249-S254) and J (Q259-L273). Three short, antiparallel $\beta$-sheets, consisting of only three residues per strand $(\mathrm{V} 1-\mathrm{C} 3$ and N8-A10; F129-L131 and I284-C286; A180-A182 and S192-F194) complete the fold. Four disulphide bonds (C3:C16, C15:C286, C35:C121 and C250:C316) are possible, but are not shown for the sake of simplicity of representation. Two hypothetical, long-range electron transfer (LRET) pathways, presumably involved in aromatic substrate oxidation, would include exposed histidine H83 (proceeding via A84 and N85 and H-bonded to distal histidine, H48) and exposed tryptophan W172 (proceeding via L173 and H-bonded to the porphyrin ring) (Martinez, 2002; Ruiz-Duenas et al., 2001).

When the predicted molecular model of Bjerkandera sp. RBP was superimposed on crystal models of $P$. chrysosporium peroxidases, more similar distances between backbone $\mathrm{C}^{\alpha}$ carbon atoms were obtained with LiP than with MnP. The root mean square (rms) distances were ca. $1.43 \AA$ ( 340 residues computed) after superimposition with LiP-H2 (PDB 1QPAA), $1.77 \AA$ (337 residues computed) with LiP-H8 (PDB 1LLP) and $2.13 \AA$ (332 residues computed) with MnP1 (PDB $1 \mathrm{MNP})$. Low values were also obtained via superimposition of RBP and predicted (non-crystallographic) molecular models of versatile peroxidases from P. eryngii. The RMS distances for RBP were $2.00 \AA$ (328 residues computed) after superimposition with MnPL1 (sometimes referred to as VP-PL) (PDB 1A20) and $1.81 \AA$ (337 residues computed) with PS1 (PDB 1QJR).

\section{Discussion}

The molecular mass calculated from the deduced amino acid sequence and including the carbohydrate content, is ca. $37 \mathrm{kDa}$; this figure is in good agreement with that obtained via mass spectrometry. The discrepancy value obtained via SDS-PAGE could arise because of interference by the carbohydrate moiety; it has been suggested (Matagne et al., 1991) that a very large negative charge at neutral $\mathrm{pH}$ is responsible for anomalous behaviour of proteins in SDS/PAGE.

The experimental isoelectric point $(\mathrm{p} I)$ of 3.5 is significantly different from the value 4.3 derived from the amino acid sequence; this realisation may be related to posttranslational processing, namely glycosylation, as described elsewhere (Gianazza, 1995). The abundance of acidic residues in the RBP amino acid sequence 
(12.4\% versus $4.4 \%$ of basic residues) is consistent with such a low $\mathrm{p} I$.

The rbpa nucleotide sequence revealed $98 \%$ identity with the cDNA over 1017 nucleotides. Two residues are different between the proteins translated from the cDNA and the rbpa gene; both changes, T167 in the cDNA to I167 in the rbpa gene and L301 to S301, respectively, are located in highly variable zones arising in fungal peroxidases. The primary structures of several fungal peroxidases contain either threonine or isoleucine at position 167, exactly at the beginning of $\alpha$-helix F, without any effect on the secondary and tertiary structures of the protein. Furthermore, leucine or serine are both likely to be present at position 301 . These results strongly suggest that both sequences might encode two very similar proteins (i.e. bearing more than $99 \%$ identity), most likely encoded by two allelic forms of the same gene. The work discussed hereafter relates only to the rbpa gene.

The rbpa orf has a high codon usage bias, especially in the third codon base that is likely correlated to high gene expression, as detected during enzyme purification. RBP was the most abundant peroxidase produced and was secreted into the extracellular medium by Bjerkandera sp. B33/3.

The mature RBP is preceded by a 27 amino acidleader sequence, characterised by a molecular mass of $2.9 \mathrm{kDa}$. Prediction of this signal peptidase cleavage site (Nielsen et al., 1999) suggests that RBP is synthesised as a preproenzyme, encompassing a 22 amino acid-prepeptide followed by a 5 amino acid-propeptide (Gold and Alic, 1993). The 22 amino acid-N-terminus of the RBP signal prepeptide consists of a positively charged region of 4 residues, following the initiation methionine residue (MAFKQ, with a net charge of +1 ) and of a hydrophobic region of 12 residues (LLTAALSIALAL), followed by a neutral but polar region of 5 residues (PFSQA). The five-residue propeptide (AITRR) possesses a cleavage site after a dibasic site consisting of two arginine residues; this indicates that its removal might involve an endoprotease belonging to the serine protease family. Many secreted proteins contain similar propeptides, which might be important for accurate cleavage by signal peptidases (Gold and Alic, 1993). Possible roles of propeptides involve protein targeting, polypeptide folding or enzyme maintaining in an inactive state during translocation. The RBP signal peptide might thus be cleaved during transit into the endoplasmic reticulum, whereas its propeptide cleavage probably occurs in the trans-Golgi complex (Gold and Alic, 1993). However, the actual biological significance of the propeptide remains to be elucidated.

Signal peptides are known to control the entry of eukaryotic proteins into the secretory pathway. RBP has all typical characteristics of a secreted protein, in agreement with the fact that it was first isolated in the extracellular fluid.

One potential $N$-glycosylation residue (NMT), eight cysteine residues and heme pocket residues (R44, F47, H48, E79, N85, H177, F194 and D239) including both distal and proximal histidines H48 and H177, were detected. Potential calcium binding residues (D49, G67, D69, S71, S178, D195, I197, I200 and D202), as well as residues involved in substrate oxidation (E37, E41 and D183 for manganese and H83, F149, W172 and A240 for aromatic substrates) are also present (Banci, 1997; Martinez, 2002; Ruiz-Duenas et al., 2001). This further confirms that the previously isolated enzyme, as well as that encoded by the rbpa gene is a versatile peroxidase (Martinez, 2002; Ruiz-Duenas et al., 2001), which bears the ability to oxidise both manganese and aromatic substrates.

On the basis of homology with cDNA and using the GT-AG rule of the intron splice sequences, it was possible to predict the intron/exon structure of the $1771 \mathrm{bp}$ rbpa gene.

The consensus sequences for fungal introns, $5^{\prime}$ GTDNNN, YAG-3', is highly conserved in the $1771 \mathrm{bp}$ fragment, as already observed by Kimura et al. (1991) for the LiP gene of B. adusta. As happens in other filamentous fungi, all introns of the rbpa gene contain a NNHTNAY sequence close to the $3^{\prime}$-splicing site, which is related to the yeast TACTAAC sequence. Furthermore, the putative internal Lariat fully agrees with the NNHTNAY rule. Few introns of filamentous fungi follow exactly the yeast consensus, but most have CT at positions 4 and 5 of the element and A at position 6 , with the consensus abiding to the aforementioned rule (Unkles, 1992).

A previous study (Camarero et al., 2000) revealed that several groups of genes can be established (according to the intron positions) including genes coding for peroxidases of the same type (MnP, LiP or VP) isolated from the same genus. In fact, the MnP- and LiPencoding genes from $P$. chrysosporium exhibit very different intron positions and this is also the case in 
Bjerkandera sp. genes (Fig. 2). The intron positions in fungal peroxidases presented in Fig. 2 emphasize that the rbpa gene is highly similar to the P. chrysosporium LiPEH8-, LiPH8- and LiPH10-encoding genes.

In the dendogram that compares 38 sequences of mature fungal peroxidases (Fig. 4), the $C$. cinereus enzyme appeared unrelated to the white-rot fungi peroxidases. The T. versicolor manganese-repressed peroxidase (MRP) and the G. applanatum MnP1 form the well-separated (strongly supported by bootstrapping) Group V cluster. Analysis of the G. applanatum MnP1 sequence indicates that it might possess a long range hypothetical electron transfer pathway beginning at W172, thus revealing a new possible versatile peroxidase. Within the remainder of the white-rot peroxidases, the $P$. chrysosporium, $C$. subvermispora, $P$. radiate and $D$. squalens $\mathrm{MnPs}$ are clustered together in group I, which is separated from the other ligninolytic enzymes. The LiP clade (Group IV) is backed up by a very weak support, with a bootstrap value of $35 \%$, although the internal clades that separate the Trametes and Phanerochaete LiPs are strongly supported by 97 and $100 \%$ bootstrap values, respectively. All Pleurotus sp. peroxidases including the versatile peroxidases, are clustered in Group II with $L$. irina VPS1, a putative versatile peroxidase (Zorn et al., 2003). Groups II and III are strongly supported in this dendogram, with 83 and $87 \%$ bootstrap values, respectively. Furthermore, it is possible to describe a versatile peroxidase broader clade (VP), that includes both Groups II and III, as already described by Martinez (2002) and which is supported by bootstrapping with a slightly lower score $(70 \%)$.

Well-characterised versatile peroxidases appear as two separate groups (Groups II and III); Pleurotus versatile peroxidases appear together, whereas RBP is associated with manganese peroxidases and other undefined peroxidases from Trametes sp. (not clustered in Group I), such as a $\mathrm{MnP}$ from $P$. radiate.

At present, it is not possible to assess the presence of multiple genes coding for RBP in Bjerkandera, even though the differences between cDNA and DNA sequences might indicate so.

The overall topology of such globular proteins as peroxidases is usually characterised by polypeptide segments ( $\alpha$-helices and $\beta$-strands) linked by tight turns or bends, which are almost always on the molecule surface. A three-dimensional model for the mature protein RBP (Fig. 5) was obtained by homology modelling. Its main characteristics are similar to those of fungal peroxidase models, especially those of versatile peroxidases.

The most important feature of the molecular model is that it provides a structural basis to explain the catalytic properties of RBP. Its ability to oxidize manganese with high substrate affinity is related to the presence of a manganese-binding site, that enables oxidation of this cation by the internal heme moiety (Banci, 1997; Martinez, 2002; Ruiz-Duenas et al., 2001). The two residues, H83 and W172, located on the protein surface could be involved in oxidation of aromatic substrates. It is noteworthy that three aromatic residues (F47, F159 and F165) are close to one another and could also play a role in the LRET (Martinez, 2002; Ruiz-Duenas et al., 2001).

When the predicted molecular model of RBP was superimposed onto crystal models of $P$. chrysosporium peroxidases, more similar distances between backbone $\mathrm{C}^{\alpha}$ carbons were obtained with LiP than with $\mathrm{MnP}$. These results are also in agreement with those obtained from the intron and sequence analysis.

Overall, our results indicate that the enzyme produced by the Bjerkandera sp. strain B33/3, of which some biochemical and molecular characteristics were conveyed in this communication, can be included, together with those produced by B. adusta and Pleurotus sp., in a new class of versatile peroxidases that share catalytic properties with both LiP and MnP.

\section{Acknowledgements}

P. Moreira is a doctoral fellowship holder (PRAXIS XXI/BD/15825/98), granted by FCT (Portugal). C. Duez is a 'Chercheur Qualifié', funded by Fonds National de la Recherche Scientifique (Belgium). The work in Liège was specifically supported by the Belgian Programme on Interuniversity Poles of Attraction (PAI No. P05/33), funded by Services Fédéraux des Affaires Scientifiques, Techniques et Culturelles.

\section{References}

Altschul, S.F., Madden, T.L., Schäffer, A.A., Zhang, J., Zhang, Z., Miller, W., Lipman, D.J., 1997. Gapped BLAST and PSI- 
BLAST: a new generation of protein database search programs. Nucleic Acids Res. 25, 3389-3402.

Banci, L., 1997. Structural properties of peroxidases. J. Biotechnol 53, 253-263.

Bernstein, H., 2003. Program RasMol Molecular Rendering. Distributed by author.

Biosym Technologies I., 1993a. Program InsightII.

Biosym Technologies I., 1993b. Program Discover.

Burkert, U., Allinger, N.L., 1982. Molecular Mechanics. American Chemical Society, Washington, DC, USA.

Camarero, S., Bockle, B., Martinez, M.J., Martinez, A.T., 1996. Manganese-mediated lignin-degradation by Pleurotus pulmonarius. Appl. Environ. Microbiol. 62, 10701072.

Camarero, S., Sarkar, S., Ruiz-Duenas, F.J., Martinez, M.J., Martinez, A.T., 1999. Description of a versatile peroxidase involved in the natural degradation of lignin that has both manganese peroxidase and lignin peroxidase substrate interaction sites. J. Biol. Chem. 274, 10324-10330.

Camarero, S., Ruiz-Duenas, F.J., Sarkar, S., Martinez, M.J., Martinez, A.T., 2000. The cloning of a new peroxidase found in lignocellulose cultures of Pleurotus eryngii and sequence comparison with other fungal peroxidases. FEMS Microbiol. Lett. 191, 37-43.

Felsenstein, J., 1993. Phylogeny Inference Package (PHYLIP). Distributed by author.

Finzel, B.C., Poulos, T.L., Kraut, J., 1984. Crystal structure of yeast cytochrome $C$ peroxidase refined at $1.7 \AA$ resolution. J. Biol. Chem. 259, 3027-3036.

Gianazza, E., 1995. Isoelectric focusing as a tool for the investigation of posttranslational processing and chemical modifications of proteins. J. Chromatogr. A 705, 6787.

Giardina, P., Palmieri, G., Fontanella, B., Rivieccio, V., Sannia, G., 2000. Manganese peroxidase isoenzymes produced by Pleurotus ostreatus grown on wood sawdust. Arch. Biochem. Biophys. 376, 171-179.

Gold, M.H., Alic, M., 1993. Molecular biology of the lignin degrading basidiomycete Phanerochaete chrysosporium. Microbiol. Rev. 57, 605-622.

Guex, N., Peitsch, M.C., 1997. Swiss-model and the SwissPdbViewer: an environment for comparative protein modelling. Electrophoresis 18, 2714-2723.

Hall, T.A., 1999. BioEdit: a user-friendly biological sequence alignment editor and analysis program for Windows 95/98/NT. Nucleic Acids Symp. Ser. 41, 95-98.

Heinfling, A., Martinez, M.J., Martinez, A.T., Bergbauer, M., Szewzyk, U., 1998a. Purification and characterization of peroxidases from the dye-decolorizing fungus Bjerkandera adusta. FEMS Microbiol. Lett. 165, 43-50.

Heinfling, A., Martinez, M.J., Martinez, A.T., Bergbauer, M., Szewzyk, U., 1998b. Transformation of industrial dyes by manganese peroxidases from Bjerkandera adusta and Pleurotus eryngii in a manganese-independent reaction. Appl. Environ. Microbiol. 64, 2788-2793.

Heinfling, A., Ruiz-Duenas, F.J., Martinez, M.J., Bergbauer, M., Szewzyk, U., Martinez, A.T., 1998c. A study on reducing substrates of manganese-oxidizing peroxidases from Pleurotus eryngii and Bjerkandera adusta. FEBS Lett. 428, 141146.

Kimura, Y., Asada, Y., Oka, T., Kuwahara, M., 1991. Molecular analysis of a Bjerkandera adusta lignin peroxidase gene. Appl. Microbiol. Biotechnol. 35, 510-514.

Martinez, A.T., 2002. Molecular biology and structure-function of lignin degrading heme peroxidases. Enzyme Microb. Technol. 30, 425-444.

Matagne, A., Joris, B., Frere, J.M., 1991. Anomalous behaviour of a protein during SDS/PAGE corrected by chemical modification of carboxylic groups. Biochem. J. 280, 553556.

Mester, T., Field, J.A., 1998. Characterization of a novel manganese peroxidase-lignin peroxidase hybrid isozyme produced by Bjerkandera species strain BOS55 in the absence of manganese. J. Biol. Chem. 273, 1541215417.

Moreira, P.R., Almeida-Vara, E., Sena-Martins, G., Polonia, I., Malcata, F.X., Duarte, J.C., 2001. Decolourisation of Remazol Brilliant Blue R via a novel Bjerkandera sp. strain. J. Biotechnol. 89, 107-111.

Nielsen, H., Brunak, S., von Heijne, G., 1999. Machine learning approaches for the prediction of signal peptides and other protein sorting signals. Protein Eng. 12, 3-9.

Palma, C., Martinez, A.T., Lema, J.M., Martinez, M.J., 2000. Different fungal manganese-oxidizing peroxidases: a comparison between Bjerkandera sp. and Phanerochaete chrysosporium. J. Biotechnol. 77, 235-245.

Peitsch, M.C., 1995. Protein modeling by e-mail. Biotechnology 13, 658-660.

Peitsch, M.C., 1996. ProMod and Swiss-model: internet-based tools for automated comparative protein modelling. Biochem. Soc. Trans. 24, 274-279.

Pitcher, D.G., Saunders, N.A., Owen, R.J., 1989. Rapid extraction of bacterial genomic DNA with guanidium thiocyanate. Lett. Appl. Microbiol. 8, 151-156.

Ruiz-Duenas, F.J., Martinez, M.J., Martinez, A.T., 1999a. Heterologous expression of Pleurotus eryngii peroxidase confirms its ability to oxidize $\mathrm{Mn}^{2+}$ and different aromatic substrates. Appl. Environ. Microbiol. 65, 47054707.

Ruiz-Duenas, F.J., Martinez, M.J., Martinez, A.T., 1999b. Molecular characterization of a novel peroxidase isolated from the ligninolytic fungus Pleurotus eryngii. Mol. Microbiol. 31, 223235.

Ruiz-Duenas, F.J., Camarero, S., Perez-Boada, M., Martinez, M.J., Martinez, A.T., 2001. A new versatile peroxidase from Pleurotus. Biochem. Soc. Trans. 29, 116-122.

Shevchenko, A., Chernushevich, I., Wilm, M., Mann, M., 2000. De novo peptide sequencing by nanoelectrospray tandem mass spectrometry using triple quadrupole and quadrupole/time-of-flight instruments. In: Chapman, J.R. (Ed.), Protein and Peptide Analysis. Humana Press, Totowa, NJ, pp. 1-16.

Unkles, S.E., 1992. Gene organization in industrial filamentous fungi. In: Kinghorn, J.R., Turner, G. (Eds.), Applied Molecular 
Genetics of Filamentous Fungi. London University Press, Cambridge, pp. 28-53.

Weiner, S., Kollman, P.A., 1981. AMBER: assisted model building with energy refinement. A general program for modeling molecules and their interactions. J. Comp. Chem. 2, 287303.
Weiner, S., Kollman, P.A., Nguyen, D.T., Case, D.A., 1986. An all atom force field for simulations of proteins and nucleic acids. J. Comp. Chem. 7, 230-252.

Zorn, H., Langhoff, S., Scheibner, M., Nimtz, M., Berger, R.G., 2003. A peroxidase from Lepista irina cleaves beta-carotene to flavor compounds. Biol. Chem. 384, 1049-1056. 